\title{
Prevalence and symptomatology of polycystic ovarian syndrome in Indian women: is there a rising incidence?
}

\author{
Anjali Choudhary*, Shweta Jain, Priyanka Chaudhari
}

Department of Obstetrics and Gynecology, Sri Guru Ram Rai Institute of Medical and Health Services, Patel Nagar, Dehradun, Uttarakhand, India

Received: 20 August 2017

Accepted: 16 September 2017

*Correspondence:

Dr. Anjali Choudhary,

E-mail: mcgsunil@gmail.com

Copyright: () the author(s), publisher and licensee Medip Academy. This is an open-access article distributed under the terms of the Creative Commons Attribution Non-Commercial License, which permits unrestricted non-commercial use, distribution, and reproduction in any medium, provided the original work is properly cited.

\section{ABSTRACT}

Background: Polycystic ovarian syndrome [PCOS] is a complex ill understood hormonal disorder resulting in myriads of symptoms like irregular menses, hyperandrogenism, metabolic disturbances and infertility. It occurs in about $8-11 \%$ of reproductive age group women. Recently changing lifestyles and rising obesity worldwide have contributed to rise in the incidence of PCOS. Objective of the present is to study the prevalence and symptomatology of PCOS in Indian women.

Methods: 170 women in reproductive age group, with irregular menstrual cycles attending OPD, voluntarily participated in a cross sectional study. All were subjected to detailed history, examination and investigated with a battery of lab tests to confirm PCOS. Out of 170 women investigated 70 women with features of PCOS were included in the study (diagnosed as PCOS by NIH criteria).

Results: The overall prevalence of PCOS in the study population was, $41 \%$. It was $16 \%$ in married women and $24 \%$ in unmarried girls. Common menstrual irregularity was-oligomenorrhea (40\%), Menorrhagia (12.8\%) amenorrhea (11\%). Common symptoms were hirsutism, acne, infertility and alopecia.

Conclusions: PCOS is rising in young women and to some extent the changing lifestyle in urban women may be linked to it. There in a world-wide increase in the incidence of obesity, diabetes mellitus and metabolic syndrome, PCOS and its accompanying insulin resistance is contributing to it. Treating PCOS and its complications is adding to health care burden.

Keywords: Changing lifestyle, PCOS, Prevalence, Rising incidence, Symptomatology

\section{INTRODUCTION}

Polycystic ovarian syndrome is a complex endocrine disorder occurring at a frequency of $11.2 \%$ in women of reproductive age group, out of which prevalence of PCOS in teenage girls may be as high as $50 \% .{ }^{1}$ It is the commonest cause of menstrual irregularities and infertility. ${ }^{2}$ First described by Stein and Levinthal in 1935 it has taken a form of a definite clinical entity. It is an ill understood mysterious condition with no definite cure. Although anovulation is the main feature, sometimes spontaneous ovulation and conception may occur. Majority of women present with irregular periods but women with regular menses may have anovulation and PCOS. Young adolescent girls experience full range of symptoms from irregular menses, amenorrhea, menorrhagia, hirsutism, acne, skin pigmentation, alopecia, ovarian cysts. Other symptoms like anxiety, depression, thyroid problems and galactorrhea, may exist. Obesity or propensity to weight gain is a common feature, though it is not uncommon in non obese women. Characterized by chronic an ovulation, 
hyperandrogenism, hormonal imbalance and metabolic disorder, it has an unclear origin. Basic pathology seems to be an increased insulin resistance which in turn causes an increased $\mathrm{LH} / \mathrm{FSH}$ ratio, decreased $\mathrm{SHBG}$, resulting in anovulation and hyperandrogenism. ${ }^{3-5}$ Insulin resistance can lead to hyperinsulinism or hyperinsulinemia. It is also a precursor to type 2 diabetes. Insulin resistance and its association with hormonal imbalance at the bio-molecular level is unclear and difficult to prove. $75 \%$ women with PCOS have insulin resistance and $10 \%$ of these women will develop Diabetes by the age 40 . Women with PCOS have a higher prevalence of MBS than the general population. Metabolic syndrome (MBS) is a combination of cardiovascular disease risk factors associated with population. Hyperandrogenemia is a risk factor for MBS independent of obesity and insulin resistance, (IR), glucose intolerance, dyslipidemia, hypertension and central obesity. ${ }^{1,2}$

Many ways of diagnosing PCOS have been described using various criteria. The NIH criteria defines PCOS as unexplained hyper androgenic anovulation, oligomenorrhea and hyperandrogenism ,either clinical or biochemical after excluding other causes of anovulation like hypothyroidism and hyperprolactinamia. ${ }^{6}$ Rotterdam criteria decided in 2003 includes two of the following features to diagnose PCOS; oligo or anovulation, clinical /biochemical signs of hyperandrogenism, polycystic ovaries. ${ }^{4}$ If Polycystic Ovarian Syndrome and therefore Insulin Resistance are missed, serious complications can develop as the adolescent matures, may even be a cause for adult metabolic syndromes., ${ }^{1,3}$ But with proper management Insulin Resistance can be reversed. There is a worldwide increase in obesity in women due to changing lifestyles and eating habits. Since adiposity is closely associated with hyperandrogenism there is a proportionate rise in PCOS. India has seen rise in the incidence of PCOS in young girls in recent years which is a direct precursor of anovulatory infertility and diabetes in the later life. ${ }^{5}$

The objectives of the present survey were to study prevalence and symptomatology of PCOS in urban young women of Uttarakhand.

\section{METHODS}

A population based cross sectional survey of 170 women in reproductive age, attending POD was conducted to detect prevalence of PCOS. All were subjected to detailed history and examination to detect PCOS. Out of these 70 women diagnosed as PCOS by NIH criteria were included in the study. All participants were subjected to a detailed questionnaire which included personal details, height, weight, age of menarche, menstrual history, history of symptoms like menstrual irregularity, hirsutism, acne, obesity or increasing weight, stria, alopecia, skin pigmentation.
Thorough physical examination to look for goiter, galactorrhea, hirsutism, pigmentation and striae was done. Height, weight and waist line was measured and BMI was calculated for all women. An enquiry was done in women's dietary habits, craving for carbohydrate rich foods, lifestyle and exercise.

The diagnostic criteria for PCOS were irregular menses, hyperandrogenism-with central obesity, hirsutism and acne-and exclusion of other cause of anovulation like hypothyroidism, hyperprolactinamia, and hyper adrenalism (The NIH criteria).

Women with irregular cycles with hypothyroidism or hyper-prolactinemia were excluded from the study

Lab studies: Lab tests included TSH, Prolactin, FSH, LH, DHEAS, free testosterone, Fasting sugar, fasting plasma insulin, and pelvic ultrasound for uterus and ovaries.

\section{RESULTS}

Results thus obtained were tabulated and analyzed.70 women fitting the NIH criteria were included in the study. Mean age was -24 years ( \pm 7 yrars), $28 \%$ were married, $40 \%$ unmarried. Women's demographic profile is listed in table 1. Overall prevalence of PCOS in women with irregular periods was $40 \%$. Prevalence in study population was $16.2 \%$ in the married women and $24 \%$ in the unmarried women.

Table 1: Demographic factors (Total No of women 70).

\begin{tabular}{|lc|}
\hline A ge group & No $(\%)$ \\
\hline $16-20$ & $25(21.4 \%)$ \\
\hline $21-25$ & $26(37.1 \%)$ \\
\hline $25-30$ & $17(24.28 \%)$ \\
\hline $30+$ & $2(2.85 \%)$ \\
\hline Urban/rural & \\
\hline Urban & $55(78 \%)$ \\
\hline Rural & $15(21.4 \%)$ \\
\hline Marital status & \\
\hline Married & $28(40 \%)$ \\
\hline Un married & $42(60 \%)$ \\
\hline
\end{tabular}

Clinically irregular menses was the commonest complaint $68 \%$, followed by; hirsutism $64 \% .58 \%$ of women had oily skin and acne -suggestive of androgenic activity. Commonest menstrual irregularity was oligomenorrhea (40\%), followed by, menorrhagia (12.8\%) amenorrhea (11\%).

Majority of our subjects were unmarried emphasizing the fact that PCOS is a common cause of irregular period in young unmarried women. $38 \%$ of these women were obese on diet of high carbohydrate content highlighting the changed eating habits of youngsters. Rest of 
symptomatoloy we observed is enlisted in the table (Table 2).

Table 2: Clinical features (Total no. 70).

\begin{tabular}{|lll|}
\hline Clinical feature & Number & Percentage* \\
\hline Irregular menses & 48 & 68 \\
\hline Obesity & 38 & 54 \\
\hline Acne & 41 & 58.57 \\
\hline Hirsutism & 45 & 64.2 \\
\hline Alopecia & 18 & 25 \\
\hline Pigmentation (acanthosis) & 21 & 30 \\
\hline Striae & 28 & 40 \\
\hline Oily skin & 40 & 57.14 \\
\hline Infertility & 28 & 40 \\
\hline
\end{tabular}

*Many women had a combination of symptoms present

On questioning about dietary habits and exercise pattern majority of obese and Normal weight women reported carbohydrate craving, sedentary life with minimal or no exercise.

Table 3: Investigations.

\begin{tabular}{|lllll|}
\hline Parameter & Normal & no $\%$ & Abnormal & no $\%$ \\
\hline $\begin{array}{l}\text { Serum } \\
\text { TSH* }\end{array}$ & 70 & $100 \%$ & - & \\
\hline Prolactin* & 70 & $100 \%$ & - & \\
\hline $\begin{array}{l}\text { FSH/LH } \\
\text { ratio** }\end{array}$ & 22 & $31.42 \%$ & 48 & $68.57 \%$ \\
\hline FSH & 70 & $100 \%$ & - & \\
\hline USG & 21 & $30 \%$ & $\begin{array}{l}49(\mathrm{~F} / \mathrm{O} \\
\text { PCOS) }\end{array}$ & $70 \%$ \\
\hline
\end{tabular}

*Normal levels of serum TSH and Prolactin in these women excluded other causes of anovulation other than PCOS.

** FSH, LH was done on Day 2 of Menstrual cycle.

Lab results of these women showed normal levels of serum TSH and Prolactin (Ruling out hypothyroidism and hyperprolactinemia as cause of anovulation) Day two FSH/LH ratio was abnormal in $68 \%$ women.

In $30 \%$ of patients ulitrasonography was normal and $40 \%$ of women showed enlarged ovaries with peripherally situated follicle suggestive of PCOS.

Laboratory testing for Fasting blood sugar and fasting plasma insulin revealed normal levels in $80 \%$ of women, suggesting that even though the underlying factor for development of hormonal imbalance and PCOS is considered as insulin resistance, it difficult to prove by laboratory tests.

\section{DISCUSSION}

Immaturity of hypothalamic-pituitary-ovarian axis in young girls immediately after menarche causing anovulation due to suboptimal FSH and LH production usually causes irregular menses. This state corrects on its own after few years resulting into regular ovulatory cycles as the $\mathrm{H}-\mathrm{P}-\mathrm{O}$ axis matures. ${ }^{1,2}$ In some young girls however anovulation persists and continues as chronic anovulation. The resulting hyperestrogenism, leads to hyperandrogenism, manifesting clinically as irregular menses, amenorrhea, menorrhagia, hirsutism, acne, oily skin, alopecia, obesity or a propensity for weight gain and multiple unruptured follicles in ovaries, collectively known as polycystic ovarian syndrome.

Diagnosis is recommended by NIH L 1999 criteria or Rotterdam criteria, with polycystic ovaries, androgen excess and an ovulation (any two criteria) in our observation from above study there is a rising incidence of PCOs in young urban women. $.^{3,4,5}-24 \%$ in young girls. Bronstein $\mathrm{J}$, et al found that not only there is a rising incidence but PCOs may be occurring at a younger age than previously thought. ${ }^{6}$ Changing lifestyle, eating habits, lack of physical exercise, increased incidence of adolescent obesity and rising stress levels may be responsible. ${ }^{7-9}$

In a study of adolescent girls Andrea D. Coviello, et al found that Adolescent girls with PCOS have a higher prevalence of metabolic syndrome than general adolescent girls with 3.8 times increased risk. Hyperandrogenamia in these girls is responsible for metabolic syndrome independent of obesity and insulin resistance. In recent years India has witnessed a 30\% rise in the incidence of PCOS in young adolescent girls due to changes in lifestyle.

Overall incidence quoted in literature is $11 \%$ of reproductive age group women but in adolescents it may be as high as $50 \%$, according to a study conducted in US. ${ }^{1}$ In present study we found the prevalence of PCOS to be $16.4 \%$ in married women and $24 \%$ in unmarried women. According to Beena Joshi, et al in a study of 768 adolescent girls the incidence of PCOS was $22.5 \%$, comparable to our results. ${ }^{10}$

In a study by Nidhi R et al Prevalence of PCOS in Indian adolescents was found to be $9.13 \%$ commonest presentation of PCOS is irregular menses. ${ }^{7}$ In present study of 170 women with menstrual irregularity we found $40 \%$ of women with PCOS this finding is in concurrence with other studies. ${ }^{10,11}$ The full blown polycystic ovarian syndrome in young may persist in adulthood leading to anovulatary subfertility and metabolic disorder. ${ }^{11,12}$

PCOS is increasingly associated with obesity and the obesity epidemic worldwide has been linked to an increased prevalence of the condition. ${ }^{2}$ We observed $38 \%$ of young women with obesity or propensity to weight gain. PCOS is also observed to be occurring at much younger age than traditionally believed and pre teen girls have reported to have insulin resistance, which is a precursor of PCOS at a later age. ${ }^{3}$ In present study there were $24 \%$ unmarried girls diagnosed with PCOS, most of these were teenagers. PCOS can cause obesity with male type of fat distribution due to hyperandrogenism and obesity on the other hand causes hyperandrogenism due 
peripheral conversion of estrogen to androgens causing anovulation and PCOS. Yiddish BO et al in their study of Impact of obesity on incidence of PCOS found that the prevalence rates of PCOS in underweight, normal-weight, overweight, and obese women were 8.2, 9.8, 9.9, and $9.0 \%$, respectively. This study concludes that obesity minimally increases risk of PCOS. ${ }^{2}$

In present observation the percentage of obese women was $38.5 \%$ and non obese were $49 \%$. Legro RS states in his chapter on evaluation of PCOS that it is increasingly associated with obesity and the obesity epidemic worldwide has been linked to an increased prevalence of PCOS. ${ }^{3}$ The condition however is not uncommon in non obese, or underweight women, we found $14 \%$ of subjects were normal weight. The link between obesity and PCOS is further strengthened by that the facts that a lifestyle change and control of weight will reduce the symptoms of PCOS and help reverse the hormonal imbalance.

Hyper androgenism, a main feature of PCOS is responsible for the male type obesity, hirsutism, oily skin acne and alopecia. We found $64 \%$ women had varying degrees of hirsutism, 54\% with ache and $24 \%$ had alopecia. Andrea Hsu Roe quoted that majority of women (80\%) show signs of androgen excess and androgen excess plays a major role in the pathophysiology of the condition. $^{4}$

Ultra-sonographic appearance of ovaries though is a diagnostic criterion according to Rotterdam criteria, all women may not have typical polycystic ovaries. In our observation, out of 70 women screened for PCOS only 49 women had Ultrasonographic appearance of polycystic ovaries.

Rising trends in the prevalence of PCOS has become a great cause for health concern as it is increasing the incidence of metabolic syndrome [MBS], i.e. insulin resistance, diabetes and dyslipidemia at a younger age. There is a growing appreciation that adolescents are at increasing risk for type II diabetes mellitus (DM) and the MBS as the prevalence of obesity increases in this population analogous to the situation in adults. ${ }^{1-3}$ Identifying women for PCOs and timely intervention is necessary to prevent long term consequences of metabolic syndrome. ${ }^{12-14}$

India is on the verge of becoming the diabetes capital of the world and young teen age girls with PCOS are contributing to it.

\section{CONCLUSION}

PCOS is occurring more frequently in the young Indian women than before. If Polycystic Ovarian Syndrome and therefore Insulin Resistance are missed; serious complications can develop as the adolescent matures. PCOS and its attendant complications are adding to the health care burden across the world. Need of times is to recognize these young women and correct the hormonal and metabolic disorder in time to rescue them before PCOS becomes a full blown metabolic syndrome. Perhaps larger studies in different locales are needed to establish an actual rising trend.

\section{ACKNOWLEDGMENTS}

Authors would like to acknowledge and appreciate the support of the Department of Obstetrics and gynecology, the management and contribution of the patients who voluntarily participated in the study.

Funding: No funding sources

Conflict of interest: None declared

Ethical approval: The study was approved by the Institutional Ethics Committee

\section{REFERENCES}

1. Coviello AD, Legro RS, Dunaif A. Adolescent girls with polycystic ovary syndrome have an increased risk of the metabolic syndrome associated with increasing androgen levels independent of obesity and insulin resistance. J Clin Endocrinol Metabol. 2006 Feb 1;91(2):492-7.

2. Yildiz BO, Knochenhauer ES, Azziz R. Impact of obesity on the risk for polycystic ovary syndrome $\mathbf{J}$ Clin Endocrinol Metab. 2008 Jan;93(1):162-8.

3. Legro RS, Arslanian SA, Ehrmann DA, Hoeger KM, Murad MH, Pasquali R, Welt CK. Diagnosis and treatment of polycystic ovary syndrome: an Endocrine Society clinical practice guideline. J Clin Endocrinol Metab. 2013 Dec 1;98(12):4565-92.

4. Roe AH, Dokras A. The diagnosis of polycystic ovary syndrome in adolescents. Rev Obstet Gynecol. 2011;4(2):45.

5. Azziz R. Controversy in clinical endocrinology: diagnosis of polycystic ovarian syndrome: the Rotterdam criteria are premature. J Clin Endocrinol Metab. 2006 Mar; 91(3):781-5.

6. Bronstein J, Tawdekar S, Liu Y, Pawelczak M, David R, Shah B. Age of onset of polycystic ovarian syndrome in girls may be earlier than previously thought. J Pediatr Adolesc Gynecol. 2011 Feb;24(1):15-20.

7. Nidhi R, Padmalatha V, Nagarathna R, Amritanshu R. Prevalence of polycystic ovarian syndrome in Indian adolescents, J Pediatr Adolesc Gynecol. 2011 Aug; 24(4):223-7.

8. Ganie MA, Kalra S. Polycystic ovary syndrome: A metabolic malady, the mother of all lifestyle disorders in women: Can Indian health budget tackle it in future?. Indian J Endocrinol Metab. 2011 Oct;15(4):239.

9. Singh R, Yadav P, Parveen R. Polycystic ovarian syndrome a management dilemma. J South Indian Federation Obstetric Gynecol. 2012;4(3):123-5.

10. Joshi B, Mukherjee S, Patil A, Purandare A, Chauhan S, Vaidya R. A cross-sectional study of 
polycystic ovarian syndrome among adolescent and young girls in Mumbai, India, Indian J Endocrinol Metab. 2014 May-Jun;18(3):317-324.

11. Boyle JA, Teede HJ. Irregular menstrual cycles in a young woman. CMAJ. 2014 Aug 5;186(11):850-2.

12. Sirmans SM, Pate KA. Epidemiology, diagnosis, and management of polycystic ovary syndrome. Clin Epidemiol. 2014;6:1-13.

13. Salmi DJ, Zisser HC, Jovanovic L. Screening for and treatment of polycystic ovary syndrome in teenagers. Experiment Biol Medic. 2004 May;229(5):369-77.
14. Buggs C, Rosenfield RL. Polycystic ovary syndrome in adolescence. Endocrinol Metab Clin North Am. 2005 Sep;34(3):677.

Cite this article as: Choudhary A, Jain S, Chaudhari P. Prevalence and symptomatology of polycystic ovarian syndrome in Indian women: is there a rising incidence? Int $\mathbf{J}$ Reprod Contracept Obstet Gynecol 2017;6:4971-5. 